\title{
Evaluation of salivary biomarkers for the diagnosis of periodontitis
}

\author{
Yong Zhang ${ }^{1}$, Ni Kang ${ }^{2,3}$, Fei Xue ${ }^{1}$, Jing Qiao ${ }^{1}$, Jinyu Duan ${ }^{1}$, Fan Chen ${ }^{4^{*}+}$ and Yu Cai ${ }^{2,3^{*}+}$
}

\begin{abstract}
Background: Salivary interleukin (IL)-1 $\beta$, matrix metalloproteinase (MMP)-8, pyridinoline cross-linked carboxyterminal telopeptide of type I collagen (ICTP) and Porphyromonas gingivalis (Pg) are related to periodontitis. This study aimed to investigate the diagnostic potential of these biomarkers and to build a prediction panel for diagnosing periodontal disease.

Methods: A total of 80 participants were enrolled in a cross-sectional study and divided into healthy $(n=25)$, gingivitis $(n=24)$, and periodontitis $(n=31)$ groups based on their periodontal exam results. A full mouth periodontal examination was performed and unstimulated saliva was collected. Salivary IL-1 $\beta$, MMP-8, ICTP, and Pg were assessed using enzyme-linked immunosorbent assay (ELISA) and quantitative real time PCR (qPCR). Their potentials for diagnosing periodontal disease were analyzed and combined prediction panels of periodontal disease were evaluated.

Results: As a single marker, IL-1 $\beta$ showed the best diagnostic value of the four markers evaluated and exhibited an area under the curve (AUC) value of 0.88 with $90 \%$ sensitivity and $76 \%$ specificity for discriminating periodontitis subjects from healthy subjects, an AUC value of 0.80 with $83 \%$ sensitivity and $76 \%$ specificity for discriminating gingivitis subjects from healthy subjects and an AUC value of 0.66 with $68 \%$ sensitivity and $64 \%$ specificity for differentiating periodontitis subjects from gingivitis subjects. The combination of IL-1 $\beta$, ICTP, and Pg exhibited the highest efficacy for discriminating periodontitis subjects from healthy subjects $(A \cup C=0.94)$ and gingivitis subjects ( $A U C=0.77)$. The combination of IL-1 $\beta$ and MMP-8 exhibited the best ability to discriminate gingivitis from healthy subjects (AUC $=0.84$ ).
\end{abstract}

Conclusions: Salivary IL-1 $\beta$, MMP-8, ICTP, and Pg showed significant effectiveness for diagnosing periodontal disease. The combination of IL-1 $\beta, I C T P$, and Pg can be used to discriminate periodontitis subjects from healthy subjects and gingivitis subjects, and the combination of IL-1 $\beta$ and MMP-8 can be used to discriminate gingivitis subjects from healthy subjects.

Keywords: Saliva, Biomarker, Diagnosis, Periodontitis, Gingivitis

*Correspondence: navy_fun@163.com; jessonjesson@hotmail.com

†Fan Chen and Yu Cai have contributed equally to this work.

${ }^{2}$ Department of Periodontology, Peking University School and Hospital of Stomatology and National Center of Stomatology and National Clinical Research Center for Oral Diseases and National Engineering Laboratory for Digital and Material Technology of Stomatology and Beijing Key Laboratory of Digital Stomatology, No.22 South Avenue Zhongguancun, Beijing 100081, People's Republic of China

${ }^{4}$ Department of Stomatology, People's Hospital of Peking University, No.11 Beijing Xizhimen South Street, Beijing 100044, People's Republic of China

Full list of author information is available at the end of the article

\section{Background}

Periodontal disease is a chronic inflammatory disease induced by pathogenic bacteria that results in connective tissue and alveolar bone destruction [1]. Traditionally, diagnosis of periodontal disease has been based on clinical and radiographic examinations that reflect a previous history of disease but lack ability to detect current disease activity [2]. Early detection of periodontal tissue destruction can be useful to monitor a disease's progression and prevent future destruction. Saliva collection is a simple noninvasive bodily fluid 
test that can be effective for diagnosis, because saliva contains omics constituents that can reflect the current physiological status of periodontal tissue $[3,4]$.

In the past several decades, various salivary markers (bacteria [5], host enzymes [6], cytokines [7], and bone metabolic products [8]) have been investigated as targets to differentiate between periodontitis patients and healthy subjects. However, there have been inconsistent or even contrary results in previous studies [9-11]. As we know, during the progress of periodontal disease, in the gingivitis stage, patients exhibit gingival inflammation without connective tissue or bone destruction. In the early stage of periodontitis, patients display gingival inflammation with connective tissue destruction and progress to alveolar bone destruction at later stages of disease. We believe that the change of markers occurs consecutively at different phases of periodontal disease, so a biomarker combination can be more effectively used for diagnosing disease status. Salivary interleukin (IL)-1 $\beta$ [12], matrix metalloproteinase (MMP)-8 [13], pyridinoline cross-linked carboxyterminal telopeptide of type I collagen (ICTP) [14], and Porphyromonas gingivalis (Pg) [15] display high prevalence in the populations of interest and are strongly related to periodontitis. The aim of this study is to evaluate the diagnostic efficiency of these markers (representing inflammation, tissue degradation, and periodontal pathogens) among healthy, gingivitis, and periodontitis subjects and to combine them in order to build an effective prediction panel for diagnosing periodontal disease.

\section{Methods}

\section{Study design}

This study was designed and performed as a cross-sectional study. It was approved by the human subjects ethics board of Peking University School and Hospital of Stomatology and was conducted in accordance with the Helsinki Declaration of 1975, as revised in 2013. All participants were informed verbally and in writing and each provided written informed consent. All primary data were collected according to Strengthening the Reporting of Observational Studies in Epidemiology guidelines.

The inclusion criteria were: (1) 20-65 years of age, (2) having at least 20 teeth, (3) underwent no medical treatment during the last 3 months before examination and sampling (4) non-smoker, (5) no history of systemic disease.

The exclusion criteria were (1) wore orthodontic appliances, (2) pregnant or currently in the breast-feeding period, (3) having undergone periodontal therapy within the 6 months prior to the examination and sampling.

\section{Clinical evaluations}

All participants were recruited at the department of periodontology, first clinical division, Peking University School and Hospital of Stomatology. All participants received a full mouth periodontal examination and a medical and dental history evaluation by one single examiner. All permanent teeth were measured with a 10-mm manual periodontal probe (PCP10-SE, HuFriedy, Chicago, USA) and measurements were rounded upwards to the nearest millimeter. Plaque Index (PI) and bleeding on probing (BOP) were measured at 4 sites (mesial, distal, buccal, and lingual). Probing depth (PD) and clinical attachment loss (CAL) were measured at 6 sites on all teeth. BOP was considered present if the probed site bled for approximately $20 \mathrm{~s}$ after probing.

\section{Patient groups}

Based on the results of the examinations, all participants were divided into 3 groups: healthy group $(\mathrm{H})$, gingivitis group (G) and periodontitis group (P) in accordance with the 2017 World Workshop on the Classification of Periodontal and Peri-Implant Diseases and Conditions [16, 17].

1. Healthy group $(\mathrm{H})$ : Subjects with absence of bleeding on probing $(\mathrm{BOP}<10 \%)$, probing depth $(\mathrm{PD}) \leq 3 \mathrm{~mm}$, no clinical attachment loss (CAL), no radiographic bone loss, no sign of other inflammatory lesions in the oral mucosa.

2. Gingivitis group (G): Subjects with presence of bleeding on probing and $\mathrm{BOP} \geq 10 \%, \mathrm{PD} \leq 3 \mathrm{~mm}$, no clinical attachment loss, no radiographic bone loss.

3. Periodontitis group (P): Subjects with presence of interdental $C A L \geq 5 \mathrm{~mm}, \mathrm{PD} \geq 6 \mathrm{~mm}$ and radiographic bone loss extending to $2 / 3$ of the root or beyond. Participants had lost no more than 4 teeth due to periodontitis. In addition, to estimate periodontitis progression, disease stage and grade was determined by evaluating radiographic bone loss/age [18]. Radiographic bone loss was determined using the tooth showing the most severe bone loss as a percentage of root length. Since the values of \% bone loss/age were $>1.0$. All participants were considered to be in stage III grade $\mathrm{C}$ periodontitis.

\section{Saliva collection and analysis}

Saliva collection was performed according to the technique proposed by Henson et al. [19]. Participants were asked to refrain from eating, drinking, smoking or engaging in oral hygiene procedures for at least two hours prior to saliva collection. Subjects rinsed their mouths with tap water for $30 \mathrm{~s}$ approximately $10 \mathrm{~min}$ prior to saliva 
collection and then expectorated into sterile tubes while seated in an upright position. $5 \mathrm{ml}$ of unstimulated saliva samples were collected, then saliva samples were centrifuged at $5000 \mathrm{~g}$ for $5 \mathrm{~min}$ at $4{ }^{\circ} \mathrm{C}$. Supernatants were removed from the pellet. $0.5 \mathrm{ml}$ aliquots of the resultant supernatant and pellets were stored at $-80{ }^{\circ} \mathrm{C}$ until analysis.

Salivary IL-1 $\beta$, MMP-8, and ICTP levels were detected and measured using commercial enzyme-linked immunosorbent assay (ELISA) kits obtained from R\&D Systems (Minneapolis, MN, USA) and Orion Diagnostica (Espoo, Finland), according to the manufacturer's instructions. Salivary Pg DNA was extracted from pellets using UltraClean Microbial DNA Isolation Kits (MO BIO Laboratories Inc, Carlsbad, California, USA)Then, quantities of Pg were determined using quantitative real time PCR (qPCR). Primers for the 16S rRNA gene of Pg were used as follows: forward primer, $5^{\prime}$-GCGCTCAAC GTTCAGCC-3'; reverse primer, 5'-CACGAATTCCGC CTGC-3'. qPCR was performed in duplicate in reaction volumes of $10 \mu \mathrm{l}$ using Power SYBR-Green Master Mix (Applied Biosystems, Foster City, California, USA) for $15 \mathrm{~min}$ at $95.8{ }^{\circ} \mathrm{C}$ for initial denaturing, followed by 40 cycles of $95.8{ }^{\circ} \mathrm{C}$ for $30 \mathrm{~s}$ and $60.8{ }^{\circ} \mathrm{C}$ for $30 \mathrm{~s}$. Cycle Threshold $(\mathrm{Ct})$ values were calculated subsequent to this procedure.

\section{Statistical analysis}

Based on the results from Wu et al. [20], Mishra et al. [14], and Zeller et al. [21] that compared IL-1 $\beta$, MMP-8, ICTP, and Pg between periodontitis patients and healthy subjects, we assumed an equal standard deviation in the healthy, gingivitis, and periodontitis groups. G*Power 3.1.9.2 software was used to perform sample calculations, using F-test for one-way ANOVA, considering effect size of 0.4 , statistical power of $80 \%$, significance level of $95 \%$ $(\alpha<0.05)$ two-tailed. Based on this, a minimum of 22 participants were required for each group to indicate a difference between groups and this was set as the sample size requirement of the study. The SPSS statistical program (Version 21.0; SPSS Inc., Chicago, IL, USA) was used to analyze the data. The mean with standard deviation was used to describe the variables of the demographic and clinical characteristics as well as the distribution of biomarkers among the healthy, gingivitis, and periodontitis groups. Independent t-tests were used to evaluate the differences between the three groups. Correlations between biomarkers and clinical indications were assessed using a linear regression analysis and logistic regression adjusted for age and gender. These results were used to establish panels for predicting gingivitis and periodontitis according to an automatic stepwise selection strategy. Receiver operating characteristic (ROC) curve analysis and corresponding area under the curve (AUC) analyses were used to evaluate the performance of biomarkers and predictive panels. Cut-off values were obtained using the ROC curves. The sensitivity and specificity for the biomarker combinations were estimated by identifying the cut-off point of the predicted probability that yielded the highest sum of sensitivity and specificity. Statistical significance was defined as $p<0.05$.

\section{Results}

A total of 80 participants were enrolled in this study. The demographic and clinical characteristics of all participants are presented in Table 1. Gender demographics and tooth loss status were balanced among the three groups. 25 patients were placed into the healthy group $(\mathrm{H}), 24$

Table 1 Characteristics of study participants between the healthy, gingivitis, and periodontitis groups

\begin{tabular}{|c|c|c|c|}
\hline & Healthy group $(\mathrm{N}=25)$ & Gingivitis group $(\mathrm{N}=24)$ & Periodontitis group $(\mathrm{N}=31)$ \\
\hline Age & $24.68 \pm 3.52$ & $26.32 \pm 4.02$ & $42.58 \pm 3.39 * * \#$ \\
\hline Gender (M/F) & $12 / 13$ & $11 / 13$ & $17 / 14$ \\
\hline Tooth & $27.7 \pm 1.1$ & $27.6 \pm 1.2$ & $27.5 \pm 0.9$ \\
\hline Plaque index (PI) & $0.22 \pm 0.28$ & $0.76 \pm 0.58 *$ & $1.32 \pm 0.76^{*}, \#$ \\
\hline Bleeding on probing (BOP, \%) & $1.1 \pm 1.8$ & $25.8 \pm 9.7^{* *}$ & $65.1 \pm 17.1{ }^{* *} \# \#$ \\
\hline Probing depth (PD, mm) & $2.62 \pm 0.45$ & $2.95 \pm 0.29 * *$ & $4.74 \pm 0.644^{* *} \# \#$ \\
\hline Clinical attachment loss (CAL, mm) & 0 & 0 & $4.93 \pm 0.522^{* *} \# \#$ \\
\hline Unstimulated salivary flow rates (ml/min) & $0.58 \pm 0.19$ & $0.54 \pm 0.15$ & $0.56 \pm 0.14$ \\
\hline $\mathrm{IL}-1 \beta(\mathrm{pg} / \mathrm{ml})$ & $92.2 \pm 31.9$ & $128.6 \pm 33.5^{* *}$ & $162.2 \pm 55.9^{* *} \#$ \\
\hline MMP-8 (ng/ml) & $435.8 \pm 180.6$ & $603.2 \pm 220.7 *$ & $657.1 \pm 279.8^{* *}$ \\
\hline ICTP (pg/ml) & $528.8 \pm 141.1$ & $598.0 \pm 203.2$ & $789.7 \pm 246.8$ ** \#\# \\
\hline Pg (Ct value) & $13.78 \pm 1.23$ & $14.52 \pm 1.01 *$ & $15.20 \pm 1.07^{* *} \#$ \\
\hline
\end{tabular}

*Significantly different compared to healthy group $\left({ }^{*} p<0.05,{ }^{* *} p<0.01\right)$

\#Significantly different compared to gingivitis group (\#p<0.05,\#\#p<0.01) 
patients were placed into the gingivitis group $(\mathrm{G})$ and 31 patients were placed into the periodontitis group $(\mathrm{P})$. As anticipated, there were no significant differences found in the salivary flow rates between these three groups. Compared to the healthy group, the diseased (gingivitis and periodontitis) groups showed higher PI and BOP. The periodontitis group showed older, higher PD and CAL compared to healthy and gingivitis groups.

The salivary level of IL-1 $\beta$, MMP-8, ICTP, and Pg were measured and are shown in Table 1. ANOVA analysis was performed to investigate difference between the three groups. The periodontitis group showed significantly higher levels of IL-1 $\beta$, MMP- 8 , ICTP, and Pg compared to the healthy group and higher levels of IL-1 $\beta$, ICTP, and Pg compared to the gingivitis group. The gingivitis group showed higher levels of IL-1 $\beta$, MMP-8, and Pg compared to the healthy group. No significant difference in MMP-8 was found between the periodontitis and gingivitis groups; no significant difference in ICTP was found between the gingivitis and healthy groups.

We next evaluated the correlation between each marker and clinical indices (Age, PD, and BOP) using a linear regression model. The results showed that IL- $1 \beta$ and ICTP were moderately correlated with PD $(\mathrm{r}=0.43$, $0.46, p<0.01)$ and BOP $(\mathrm{r}=0.43,0.48, p<0.01)$; Pg was moderately correlated with $\mathrm{PD}(\mathrm{r}=0.41, p<0.01)$ and mildly correlated with BOP $(\mathrm{r}=0.38, p<0.01)$; MMP-8 was mildly correlated with PD $(\mathrm{r}=0.32, p<0.01)$ and BOP $(r=0.38, p<0.01)$ (Table 2). None of these four markers showed significant correlation with age, and after employing the Mann-Whitney U-test, no genderspecific differences in the levels of these markers were found $(p>0.05)$.

\section{Periodontitis group versus healthy group}

All four markers tested in this study showed a significant difference between the periodontitis and healthy groups (Table 1). IL- $1 \beta$ yielded an AUC value of 0.88 , and the other three markers yielded AUC values ranging from 0.75 to 0.85 (Table 3). Logistic regression was used to evaluate different combinations of the four biomarkers.
Among the different combinations, IL-1 $\beta$ combined with ICTP yielded a higher AUC value of 0.92 with $87 \%$ sensitivity and $80 \%$ specificity. The combination of IL- $1 \beta$, ICTP, and MMP-8 yielded an AUC value of 0.93 with $90 \%$ sensitivity and $84 \%$ specificity. The combination of IL- $1 \beta$, ICTP, and Pg yielded an AUC value of 0.94 with $94 \%$ sensitivity and $84 \%$ specificity (Fig. 1). All four biomarkers combined yielded the best AUC value of 0.95 (Table 4).

\section{Gingivitis group versus healthy group}

IL-1 $\beta$, MMP-8, and Pg levels were shown to be significantly different between the gingivitis group and the healthy group (Table 1 ). IL-1 $\beta$ and MMP- 8 yielded similar AUC values, Pg yielded a lower AUC value (Table 3). Logistic regression analysis was used to identify differences among these biomarker combinations. IL- $1 \beta$ and MMP-8 in combination yielded an AUC value of 0.84 with $92 \%$ sensitivity and $68 \%$ specificity (Fig. 1 ). The combination of IL-1 $\beta$, MMP-8, and Pg yielded a better AUC value of 0.85 , with $83 \%$ sensitivity and $80 \%$ specificity (Table 5).

\section{Periodontitis group versus gingivitis group}

IL-1 $\beta$, ICPT, and Pg levels were significantly different between the periodontitis and gingivitis groups and yielded similar AUC values (Tables 1, 3). After logistic regression analysis, among different combinations of two markers, the combination of IL- $1 \beta$ and ICTP yielded an AUC value of 0.76 with $81 \%$ sensitivity and $71 \%$ specificity, the combination of IL- $1 \beta$ and Pg yielded a similar AUC value, and the combination of IL- $1 \beta$, Pg, and ICTP yielded the best AUC value of 0.77 with $81 \%$ sensitivity and $75 \%$ specificity (Table 6, Fig. 1).

\section{Discussion}

Periodontal disease has historically been diagnosed using a patient's clinical performance in BOP, PD, and CAL tests as well as radiographic evidence of alveolar bone loss. These methods are reliable, but are also

Table 2 Correlation analysis between markers and clinical characteristics of study population

\begin{tabular}{|c|c|c|c|c|c|c|}
\hline & \multicolumn{2}{|l|}{ PD } & \multicolumn{2}{|l|}{ BOP } & \multicolumn{2}{|l|}{ Age } \\
\hline & Spearman's $\rho$ & $p$ value & Spearman's $\rho$ & $p$ value & Spearman's $\rho$ & $p$ value \\
\hline$I L-1 \beta$ & 0.428 & 0.001 & 0.433 & $<0.001$ & 0.107 & 0.560 \\
\hline MMP-8 & 0.319 & 0.008 & 0.377 & 0.002 & 0.161 & 0.187 \\
\hline ICTP & 0.458 & $<0.001$ & 0.479 & $<0.001$ & 0.167 & 0.155 \\
\hline $\mathrm{Pg}$ & 0.414 & 0.004 & 0.376 & 0.002 & 0.072 & 0.274 \\
\hline
\end{tabular}


Table 3 Diagnostic potential of salivary biomarkers between periodontitis patients and healthy subjects

\begin{tabular}{|c|c|c|c|c|c|c|c|c|}
\hline Biomarker & Cut-off value & AUC value & $95 \% \mathrm{Cl}$ & $p$ value & Sensitivity (\%) & Specificity (\%) & $\begin{array}{l}\text { Positive } \\
\text { predictive } \\
\text { (\%) }\end{array}$ & $\begin{array}{l}\text { Negative } \\
\text { predictive } \\
\text { (\%) }\end{array}$ \\
\hline \multicolumn{9}{|c|}{$\begin{array}{l}\text { Periodontitis versus Healthy ( } P \\
\text { versus } H \text { ) }\end{array}$} \\
\hline$\| \mathrm{L}-1 \beta(\mathrm{pg} / \mathrm{ml})$ & 91.1 & 0.875 & $0.785-0.965$ & $<0.001$ & 90.3 & 76.0 & 82.4 & 86.4 \\
\hline MMP-8 (ng/ml) & 594.6 & 0.761 & $0.630-0.892$ & 0.001 & 67.7 & 88.0 & 87.5 & 68.8 \\
\hline ICTP (pg/ml) & 625.0 & 0.840 & $0.727-9.953$ & $<0.001$ & 80.6 & 88.0 & 89.3 & 78.6 \\
\hline $\mathrm{Pg}$ (Ct value) & 13.81 & 0.788 & $0.668-0.908$ & $<0.001$ & 87.1 & 56.0 & 71.1 & 77.8 \\
\hline \multicolumn{9}{|c|}{$\begin{array}{l}\text { Gingivitis versus Healthy (G versus } \\
\text { H) }\end{array}$} \\
\hline $\mid \mathrm{L}-1 \beta(\mathrm{pg} / \mathrm{ml})$ & 104.6 & 0.803 & $0.671-0.935$ & $<0.001$ & 83.3 & 76.0 & 76.9 & 82.6 \\
\hline MMP-8 (ng/ml) & 583.6 & 0.766 & $0.630-0.903$ & 0.001 & 66.7 & 84.0 & 80.0 & 72.4 \\
\hline ICTP (pg/ml) & 585.0 & 0.594 & $0.435-0.754$ & 0.252 & 62.5 & 64.0 & 62.5 & 64.0 \\
\hline Pg (Ct value) & 14.17 & 0.687 & $0.534-0.840$ & 0.023 & 75.0 & 64.0 & 66.7 & 72.7 \\
\hline \multicolumn{9}{|c|}{$\begin{array}{l}\text { Peridoontitis versus Gingivitis ( } P \\
\text { versus } G \text { ) }\end{array}$} \\
\hline $\mid \mathrm{L}-1 \beta(\mathrm{pg} / \mathrm{ml})$ & 147.6 & 0.657 & $0.490-0.785$ & 0.029 & 67.7 & 64.0 & 70.0 & 61.5 \\
\hline MMP-8 (ng/ml) & 670.7 & 0.563 & $0.412-0.714$ & 0.419 & 58.1 & 52.0 & 60.0 & 50.0 \\
\hline ICTP (pg/ml) & 676.0 & 0.707 & $0.612-0.783$ & 0.002 & 77.4 & 72.0 & 77.4 & 72.0 \\
\hline Pg (Ct value) & 14.88 & 0.698 & $0.555-0.841$ & 0.011 & 67.7 & 76.0 & 77.8 & 65.5 \\
\hline
\end{tabular}

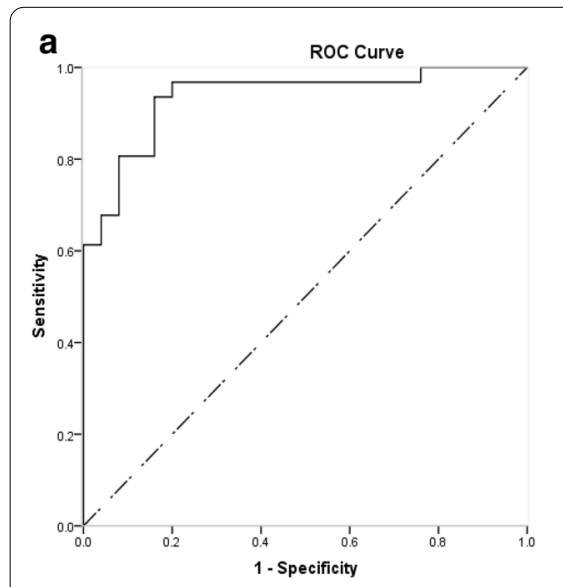

AUC: $0.9495 \% \mathrm{Cl}$ for the AUC: $0.87-1$ Sensitivity: $94 \%$ Specificity: $84 \%$

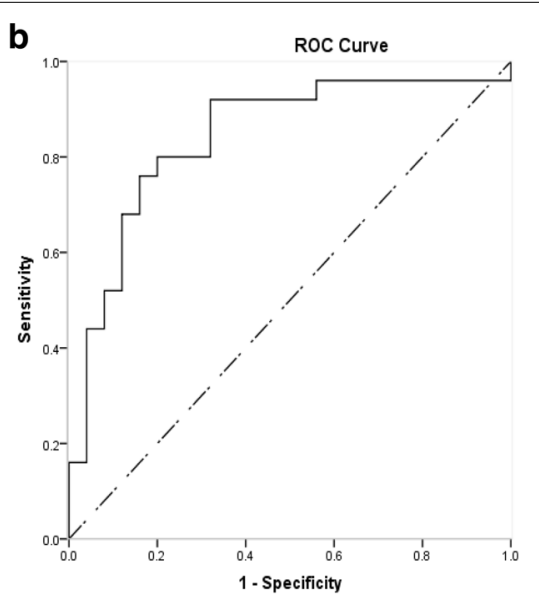

AUC: $0.8495 \% \mathrm{Cl}$ for the AUC: $0.73-0.96$ Sensitivity: $92 \%$ Specificity: $68 \%$

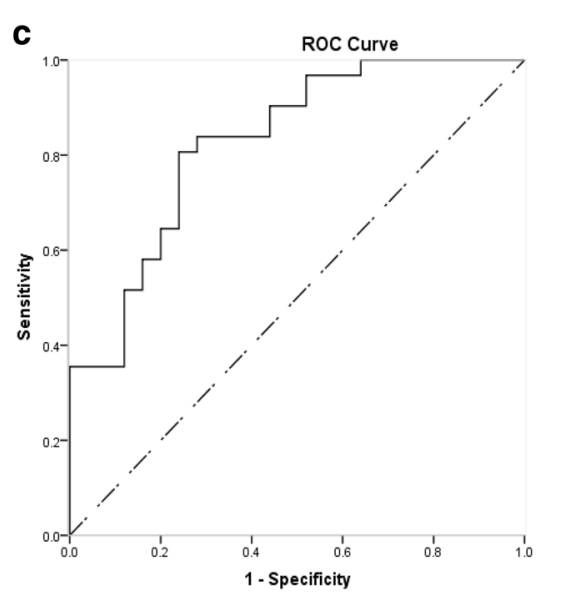

AUC: $0.7795 \% \mathrm{Cl}$ for the AUC: $0.65-0.89$ Sensitivity: $81 \%$ Specificity: $75 \%$

Fig. 1 Receiver operating characteristic (ROC) analysis of salivary biomarker combinations with AUC values: a The combination of IL-1 $\beta$, ICTP, and Pg used to discriminate periodontitis patients from healthy subjects. $\mathbf{b}$ The combination of IL-1 $\beta$ and MMP- 8 used to discriminate gingivitis patients from healthy subjects. c The combination of IL-1 1 , ICTP, and Pg used to discriminate periodontitis patients from gingivitis patients

costly and depend upon a clinician's professional experience [2]. Saliva has been proven to be a tool with a high potential value for early diagnosis and monitoring oral and systemic diseases [4, 22]. A number of salivary markers have been demonstrated to be significantly different between diseased and healthy subjects, but up to now there has been no clear and convincing biomarker that can be used for diagnosing periodontal disease. The present study proposes efficient salivary panels for diagnosing gingivitis and periodontitis and the development of saliva-based point of care (POC) technology tools that could be used in chair-side diagnostics, selfscreenings, and risk-assessment [23]. 
Table 4 Performance of salivary biomarker combinations to discriminate periodontitis patients from healthy subjects

\begin{tabular}{|c|c|c|c|c|}
\hline & Sensitivity (\%) & Specificity (\%) & AUC value & $95 \% \mathrm{Cl}$ \\
\hline$I L-1 \beta+M M P-8$ & 87.1 & 80.0 & 0.883 & $0.793-0.972$ \\
\hline$I L-1 \beta+P g$ & 87.1 & 84.0 & 0.910 & $0.828-0.991$ \\
\hline $\mathrm{IL}-1 \beta+\mathrm{MMP}-8+\mathrm{Pg}$ & 90.3 & 84.0 & 0.920 & $0.843-0.997$ \\
\hline ICTP + MMP-8 & 83.9 & 76.0 & 0.850 & $0.746-0.955$ \\
\hline $\mathrm{ICTP}+\mathrm{Pg}$ & 83.9 & 80.0 & 0.881 & $0.794-0.969$ \\
\hline IL-1 $\beta+I C T P$ & 87.1 & 80.0 & 0.917 & $0.840-0.995$ \\
\hline$I L-1 \beta+I C T P+M M P-8$ & 90.3 & 84.0 & 0.929 & $0.865-0.993$ \\
\hline $\mathrm{IL}-1 \beta+\mathrm{ICTP}+\mathrm{Pg}$ & 93.5 & 84.0 & 0.935 & $0.873-0.998$ \\
\hline$I L-1 \beta+I C T P+M M P-8+P g$ & 93.5 & 84.0 & 0.946 & $0.892-0.999$ \\
\hline
\end{tabular}

Table 5 Performance of salivary biomarker combinations to discriminate gingivitis patients from healthy subjects

\begin{tabular}{lllll}
\hline & Sensitivity (\%) & Specificity (\%) & AUC value & 95\% CI \\
\hline IL-1 $\beta+$ MMP-8 & 91.6 & 68.0 & 0.842 & $0.726-0.957$ \\
IL-1 $\beta+$ Pg & 79.2 & 76.0 & 0.819 & $0.699-0.940$ \\
IL-1 $\beta+$ MMP-8+Pg & 83.3 & 80.0 & 0.853 & $0.743-0.962$ \\
IL-1 $\beta+$ MMP-8+Pg+ICTP & 87.5 & 76.0 & 0.850 & $0.740-0.965$ \\
\hline
\end{tabular}

Table 6 Performance of salivary biomarker combinations to discriminate periodontitis from gingivitis

\begin{tabular}{|c|c|c|c|c|}
\hline & Sensitivity (\%) & Specificity (\%) & AUC value & $95 \% \mathrm{Cl}$ \\
\hline IL-1 $\beta+I C T P$ & 80.6 & 70.8 & 0.755 & $0.621-0.889$ \\
\hline$I L-1 \beta+P g$ & 77.4 & 70.8 & 0.720 & $0.584-0.856$ \\
\hline $\mathrm{ICTP}+\mathrm{Pg}$ & 74.2 & 75.0 & 0.747 & $0.618-0.876$ \\
\hline$I L-1 \beta+I C T P+P g$ & 80.6 & 75.0 & 0.770 & $0.647-0.894$ \\
\hline$I L-1 \beta+I C T P+M M P-8+P g$ & 83.9 & 70.8 & 0.770 & $0.647-0.896$ \\
\hline
\end{tabular}

We need a balance between microbial and host response to maintain periodontal health during the progress of periodontal disease. If the balance is broken, bacterial invasion, host inflammatory response, tissue and bone destructions occur non-simultaneously. After bacterial $(\mathrm{Pg})$ invasion, markers of inflammation (IL-1 $\beta$ ) are released [24]. Enzymes such as MMP-8 are produced and activated by host cells leading to the degradation of connective tissue [25], and bone degradation results in the release of ICTP into periodontal tissues and saliva [8]. Kuula's [26] and Hamedi's [27] studies revealed positive correlations between Pg infection and IL- $1 \beta$ or MMP-8 levels. Therefore, we selected these four marker candidates (IL-1 $\beta$, MMP-8, ICTP, and Pg) and evaluated their efficiency for diagnosing gingivitis and periodontitis.

IL- $1 \beta$ is a well-known inflammatory stimulator that can be used to discriminate between healthy and periodontal lesions [7]. Pg is also significantly associated with periodontal disease and has been used as a potential screening biomarker of periodontitis [28]. In this study, both of IL-1 $\beta$ and Pg showed significantly different levels among the three subject groups. Their salivary levels increased in the gingivitis group and were higher still in the periodontitis group. Our results were consistent with other studies $[15,29]$. Besides significant elevation of these biomarkers in the gingivitis and periodontitis groups, our results revealed positive correlation between $\mathrm{IL}-1 \beta, \mathrm{Pg}$, and clinical indices (PD and BOP). IL-1 $\beta$ and Pg indeed reflected periodontal status and may be valuable targets for predicting periodontal disease.

Recent studies have shown that MMP- 8 is an indicator for early periodontitis in particular [23, 30, 31]. In our study, MMP-8 was detected in significantly higher levels within the diseased groups (gingivitis and periodontitis) compared to the healthy group. However, there was no significant difference between their levels in the periodontitis and gingivitis groups. This result is in accordance with Morelli's [32] and Nascimento's [33] results. 
Verhulst [34] has also reported that MMP-8 is not associated with periodontitis. However in Heikkinen's [31] and Yucel's [35] studies, salivary MMP-8 was significantly elevated in periodontitis patients compared to gingivitis patients, and in Syndergaard's [10], Yucel's [35], and Noack's [36] studies, there were no significant difference in MMP-8 between gingivitis groups and healthy groups. These inconsistent results may be due to the evaluation of total MMP-8 in our study and Verhulst's [34] study. Sorsa's study [37] and others [38, 39] have demonstrated that total MMP-8 may not be able to effectively detect periodontal breakdown or progression of periodontitis and they concluded that, instead of total MMP-8, active MMP-8 (aMMP-8) levels truly reflect a proinflammatory state of periodontal disease. Assessment of aMMP-8 may have shown a direct correlation with periodontal disease.

Before an effective diagnosis of periodontitis, a considerable amount of alveolar bone destruction must be established. When we measured the bone loss clinically, a $2-3 \mathrm{~mm}$ threshold change was needed before exhibiting obvious destruction. This may delay diagnosis and treatment [40]. As a breakdown product of Type I collagen, ICTP is the major constituent of alveolar bone and is considered to reflect alveolar bone degradation and periodontal disease activity [41]. In our study, ICTP was not found to be significantly different between the gingivitis group and the healthy group, because there was no bone loss in these two groups. Apparent alveolar bone loss in the periodontitis group resulted in significantly higher ICTP levels compared to the gingivitis and healthy groups. This is in accordance with Mishra's [14] and Giannobile's [42] studies where they concluded that increased ICTP can be used to differentiate active gingivitis from periodontitis. Payne's [43] results have also stated that salivary ICTP concentration was significantly correlated with alveolar bone loss.

After confirming the differing biomarker results found between the periodontitis, gingivitis, and healthy groups, we examined their ability to discriminate between different periodontal clinical phenotypes. As a single marker, IL-1 $\beta$ showed the best diagnostic value of these four candidates; it exhibited an AUC value of 0.88 with $90 \%$ sensitivity and $76 \%$ specificity for discriminating periodontitis subjects from healthy subjects, an AUC value of 0.80 with $83 \%$ sensitivity and $76 \%$ specificity for discriminating gingivitis subjects from healthy subjects, and an AUC value of 0.66 with $68 \%$ sensitivity and $64 \%$ specificity for discriminating periodontitis subjects from gingivitis subjects. These are valuable results and are consistent with results from Jaedicke's [7] and Nazar's [44] investigations. These both concluded that IL- $1 \beta$ is the most robust salivary biomarker with respect to periodontal disease. Hassan's [45] results also exhibited a positive relationship between salivary IL-1 $\beta$ and gingival inflammation during pregnancy. All of these results support our inclusion of IL- $1 \beta$ as a predictive overall indicator of gingivitis and periodontitis.

Different markers may peaked at different stages over the course of disease, and when biomarkers of host and microbial origin are combined, the detection of periodontitis maybe be improved [20,46, 47]. Previous studies have pointed towards realizing a stronger discriminatory capability when IL-1 $\beta$, MMP- 8 , and other markers are combined, compared to single-marker analysis [48]. Pg and MMP-8 in combination [49], as well as ICTP and MMP-8 in combination [50], have also exhibited greater predictive value. Our data show that IL-1 $\beta$, individually, revealed an AUC value of 0.88 for discriminating periodontitis subjects from healthy subjects. The combination of IL-1 $\beta$, MMP-8, and Pg strongly improved this performance to an AUC value of 0.92 . This is consistent with Gursoy's [51, 52] results. In that study, IL-1 $\beta$, MMP-8, and $\mathrm{Pg}$ were calculated together to obtain a cumulative risk score that was highly correlated with advanced periodontitis. Although this previous study showed that biomarker combinations facilitate a more robust prediction of periodontal progression and stability, our results were different from Gursoy's [51, 52]. We demonstrated that IL- $1 \beta$ and ICTP in combination yielded a similar AUC value $(0.917)$ to differentiate periodontitis subjects from healthy subjects when compared to the combination of IL-1 $\beta$, MMP-8, and Pg (0.920), indicating that IL-1 $\beta$ and ICTP are more effective for predicting periodontitis. The combination of IL-1 $\beta$, ICTP, and Pg exhibited the best AUC value (0.94) to discriminate periodontitis subjects from healthy subjects.

As a nondestructive and reversible gingival inflammation stage, we enrolled gingivitis subjects into this study and assigned participants with more homogeneous clinical phenotypes. Our results showed that MMP-8 was not significantly elevated in the periodontitis group compared to the gingivitis group, and ICTP was not significantly elevated in the gingivitis group compared to the healthy group. This indicates that, for predicting the disease status, different marker combinations should be used to achieve an effective diagnosis. After logistic regression analysis, the combination of IL-1 $\beta$, ICTP, and Pg not only yielded the best AUC value to discriminate periodontitis patients from healthy subjects, but also exhibited the best ability to discriminate periodontitis subjects from gingivitis subjects $(\mathrm{AUC}=0.77$ ). To discriminate gingivitis from healthy subjects, IL-1 $\beta$, MMP8 , and Pg together exhibited the best AUC value of 0.85 , while IL- $1 \beta$ and MMP- 8 in combination yielded a slight lower AUC value of 0.84 . These AUC values were lower than the combination most effective at discriminating 
periodontitis subjects from healthy subjects (IL-1 $\beta$, ICTP and Pg, with an AUC of 0.94), but were above acceptable AUC values of 0.75 [53] and can potentially be used for clinical diagnosis.

However, there were some limitations in this study. We directly measured salivary concentrations of these markers, while Afacan's [54] study suggests that salivary flow rate may result in differences in saliva composition. Calibrated-total-protein salivary biomarker levels may be more effective to evaluate the diagnostic power of these biomarkers. We also evaluated the qPCR levels of total Pg in saliva and other studies have shown that P. gingivalis can be divided according to fimA genotypes; fimA type I is exclusively found in healthy subjects and fimA type II is most prevalent in periodontitis subjects $[55,56]$. We detected salivary levels of total MMP-8, however Sorsa et al.s [30, 31, 36, 37] results showed that, compared to total MMP-8, aMMP-8 was more effective at diagnosing periodontitis, so aMMP- 8 may be a more valuable biomarker of periodontal diseases. To address these limitations, larger study sample size, longitudinal studies (such as experimental gingivitis or periodontitis designs), more precise biomarker selection, and more precise detection will be required to further evaluate our selected salivary biomarkers.

\section{Conclusion}

Our study patient sample represented gingivitis and stage III periodontitis in the current classification of periodontal diseases, and our results showed that there were significant differences of salivary IL-1 $\beta$, MMP-8, ICTP, and Pg between the healthy group, gingivitis group, and stage III periodontitis group. The efficacy of several prediction panels for diagnosing gingivitis and stage III periodontitis were evaluated. We found that the combination of IL-1 $\beta$, ICTP, and Pg can be used to discriminate stage III periodontitis subjects from healthy subjects and gingivitis subjects. Further, the combination of IL- $1 \beta$ and MMP- 8 can be used to discriminate gingivitis patients from healthy subjects.

\begin{abstract}
Abbreviations

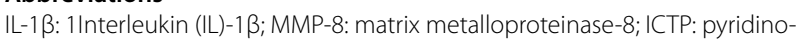
line cross-linked carboxyterminal telopeptide of type I collagen; Pg: Porphyromonas gingivalis; BOP: bleeding on probing; PI: plaque index; PD: probing depth; CAL: clinical attachment loss; ELISA: enzyme linked immunosorbent assay; qPCR: quantitative real time polymerase chain reaction; ROC: receiver operating characteristics; AUC: area under the curve; POC: point of care.
\end{abstract}

\section{Acknowledgements}

Not applicable.

\section{Authors' contributions}

Y.Z, F.C. and Y.C. wrote and edited the manuscript. Y.Z, F.C. and Y.C. conceptualized the overall strategy and developed the clinical translation and implementation. Y.Z, F.X, J.Q. and JY.D enrolled patients to the protocol, and implemented the saliva collection and processing. Y.Z, N.K and F.C. designed and performed the biomarkers measurement. Y.Z and F.C designed and performed the statistical analyses. F.C. and Y.C. were the study's principal investigators. All authors read and approved the final manuscript.

\section{Funding}

The study was supported by National Natural Science Foundations of China (Project No. 81800978) and Peking University School and Hospital of Stomatology Cao Caifang Gengyun Foundation (2017). The funders had no role in the design of the study; collection, analysis or interpretation of the data; or in writing the manuscript.

\section{Availability of data and materials}

The datasets generated during the current study are available from the corresponding author on reasonable request.

\section{Declarations}

\section{Ethics approval and consent to participate}

The Ethics Committee of the Peking University School and Hospital of Stomatology approved the study protocol (No. PKUSSIRB-2015015). All procedures performed in studies involving human participants were in accordance with the ethical standards of the institutional and/or national research committee and with the 1964 Helsinki Declaration and its later amendments or comparable ethical standards. Written informed consent for participation was obtained from each subject recruited in this study.

\section{Consent for publication}

This manuscript does not involve details, images, or videos relating to individual participants. Written informed consent of this research was obtained from all the subjects involved.

\section{Competing interests}

The authors declare that they have no conflict of interest.

\section{Author details}

${ }^{1}$ Department of First Clinical Division, Peking University School and Hospital of Stomatology and National Center of Stomatology and National Clinical Research Center for Oral Diseases and National Engineering Laboratory for Digital and Material Technology of Stomatology and Beijing Key Laboratory of Digital Stomatology, Beijing 100081, People's Republic of China. ${ }^{2}$ Department of Periodontology, Peking University School and Hospital of Stomatology and National Center of Stomatology and National Clinical Research Center for Oral Diseases and National Engineering Laboratory for Digital and Material Technology of Stomatology and Beijing Key Laboratory of Digital Stomatology, No.22 South Avenue Zhongguancun, Beijing 100081, People's Republic of China. ${ }^{3}$ Central Laboratory, Peking University School and Hospital of Stomatology and National Center of Stomatology and National Clinical Research Center for Oral Diseases and National Engineering Laboratory for Digital and Material Technology of Stomatology and Beijing Key Laboratory of Digital Stomatology, Beijing 100081, People's Republic of China. ${ }^{4}$ Department of Stomatology, People's Hospital of Peking University, No.11 Beijing Xizhimen South Street, Beijing 100044, People's Republic of China.

Received: 28 August 2020 Accepted: 29 April 2021

Published online: 17 May 2021

\section{References}

1. Slots J. Periodontitis: facts, fallacies and the future. Periodontol 2000. 2017;75(1):7-23.

2. Lang NP, Tonetti MS. Periodontal diagnosis in treated periodontitis. Why, when and how to use clinical parameters. J Clin Periodontol. 1996;23(3 Pt 2):240-50.

3. Fuentes $L$, Yakob M, Wong DT. Emerging horizons of salivary diagnostics for periodontal disease. Br Dent J. 2014;217(10):567-73.

4. Zhang Y, Sun J, Lin CC, Abemayor E, Wang MB, Wong DT. The emerging landscape of salivary diagnostics. Periodontol 2000. 2016;70(1):38-52. 
5. Belstrom D, Sembler-Moller ML, Grande MA, Kirkby N, Cotton SL, Paster BJ, Twetman S, Holmstrup P. Impact of oral hygiene discontinuation on supragingival and salivary microbiomes. JDR Clin Trans Res. 2018;3(1):57-64.

6. Ramseier CA, Kinney JS, Herr AE, Braun T, Sugai JV, Shelburne CA, Rayburn LA, Tran HM, Singh AK, Giannobile WV. Identification of pathogen and host-response markers correlated with periodontal disease. J Periodontol. 2009;80(3):436-46.

7. Jaedicke KM, Preshaw PM, Taylor JJ. Salivary cytokines as biomarkers of periodontal diseases. Periodontol 2000. 2016;70(1):164-83.

8. Frodge BD, Ebersole JL, Kryscio RJ, Thomas MV, Miller CS. Bone remodeling biomarkers of periodontal disease in saliva. J Periodontol. 2008;79(10):1913-9.

9. Teles RP, Likhari V, Socransky SS, Haffajee AD. Salivary cytokine levels in subjects with chronic periodontitis and in periodontally healthy individuals: a cross-sectional study. J Periodontal Res. 2009;44(3):411-7.

10. Syndergaard B, Al-Sabbagh M, Kryscio RJ, Xi J, Ding X, Ebersole JL, Miller CS. Salivary biomarkers associated with gingivitis and response to therapy. J Periodontol. 2014;85(8):e295-303.

11. Belstrom D, Damgaard C, Kononen E, Gursoy M, Holmstrup P, Gursoy UK. Salivary cytokine levels in early gingival inflammation. J Oral Microbiol. 2017;9(1):1364101.

12. Gursoy UK, Kononen E, Uitto VJ, Pussinen PJ, Hyvarinen K, SuominenTaipale L, Knuuttila M. Salivary interleukin-1 beta concentration and the presence of multiple pathogens in periodontitis. J Clin Periodontol. 2009;36(11):922-7.

13. de Morais EF, Pinheiro JC, Leite RB, Santos PPA, Barboza CAG, Freitas RA. Matrix metalloproteinase-8 levels in periodontal disease patients: a systematic review. J Periodontal Res. 2018;53(2):156-63.

14. Mishra D, Gopalakrishnan S, Arun KV, Kumar TS, Devanathan S, Misra SR. Evaluation of salivary levels of pyridinoline cross linked carboxyterminal telopeptide of type I Collagen (ICTP) in periodontal health and disease. J Clin Diagn Res. 2015;9(9):50-5.

15. Damgaard C, Danielsen AK, Enevold C, Massarenti L, Nielsen CH, Holmstrup P, Belstrom D. Porphyromonas gingivalis in saliva associates with chronic and aggressive periodontitis. J Oral Microbiol. 2019;11(1):1653123.

16. Chapple ILC, Mealey BL, Van Dyke TE, Bartold PM, Dommisch H, Eickholz P, Geisinger ML, Genco RJ, Glogauer M, Goldstein M, et al. Periodontal health and gingival diseases and conditions on an intact and a reduced periodontium: Consensus report of workgroup 1 of the 2017 World Workshop on the Classification of Periodontal and Peri-Implant Diseases and Conditions. J Clin Periodontol. 2018;45(Suppl 20):S68-77.

17. Papapanou PN, Sanz M, Buduneli N, Dietrich T, Feres M, Fine DH, Flemmig TF, Garcia R, Giannobile WV, Graziani F, et al. Periodontitis: consensus report of workgroup 2 of the 2017 World Workshop on the Classification of Periodontal and peri-implant diseases and conditions. J Clin Periodontol. 2018:45(Suppl 20):S162-70.

18. Tonetti MS, Greenwell H, Kornman KS. Staging and grading of periodontitis: framework and proposal of a new classification and case definition. J Clin Periodontol. 2018;45(Suppl 20):S149-61.

19. Henson BS, Wong DT. Collection, storage, and processing of saliva samples for downstream molecular applications. Methods Mol Biol. 2010;666:21-30

20. Wu YC, Ning L, Tu YK, Huang CP, Huang NT, Chen YF, Chang PC. Salivary biomarker combination prediction model for the diagnosis of periodontitis in a Taiwanese population. J Formos Med Assoc. 2018;117(9):841-8.

21. Zeller I, Hutcherson JA, Lamont RJ, Demuth DR, Gumus P, Nizam N, Buduneli N, Scott DA. Altered antigenic profiling and infectivity of Porphyromonas gingivalis in smokers and non-smokers with periodontitis. J Periodontol. 2014;85(6):837-44.

22. Dawes $C$, Wong DTW. Role of saliva and salivary diagnostics in the advancement of oral health. J Dent Res. 2019;98(2):133-41.

23. Schmalz G, Hubscher AE, Angermann H, Schmidt J, Schmickler J, Legler TJ, Ziebolz D. Associations of chairside salivary aMMP-8 findings with periodontal parameters, potentially periodontal pathogenic bacteria and selected blood parameters in systemically healthy adults. Diagn Microbiol Infect Dis. 2019;95(2):179-84.

24. Loos BG, Tjoa S. Host-derived diagnostic markers for periodontitis: do they exist in gingival crevice fluid? Periodontol. 2000;2005(39):53-72.
25. Sorsa T, Gursoy UK, Nwhator S, Hernandez M, Tervahartiala T, Leppilahti $\mathrm{J}$, Gursoy M, Kononen E, Emingil G, Pussinen PJ, et al. Analysis of matrix metalloproteinases, especially MMP-8, in gingival creviclular fluid, mouthrinse and saliva for monitoring periodontal diseases. Periodontol 2000. 2016;70(1):142-63.

26. Kuula H, Salo T, Pirila E, Tuomainen AM, Jauhiainen M, Uitto VJ, Tjaderhane $\mathrm{L}$, Pussinen PJ, Sorsa T. Local and systemic responses in matrix metalloproteinase 8-deficient mice during Porphyromonas gingivalis-induced periodontitis. Infect Immun. 2009;77(2):850-9.

27. Hamedi M, Belibasakis GN, Cruchley AT, Rangarajan M, Curtis MA, Bostanci N. Porphyromonas gingivalis culture supernatants differentially regulate interleukin-1 beta and interleukin-18 in human monocytic cells. Cytokine. 2009;45(2):99-104.

28. Liljestrand JM, Gursoy UK, Hyvarinen K, Sorsa T, Suominen AL, Kononen E, Pussinen PJ. Combining salivary pathogen and serum antibody levels improves their diagnostic ability in detection of periodontitis. J Periodontol. 2014;85(1):123-31.

29. Sexton WM, Lin Y, Kryscio RJ, Dawson DR 3rd, Ebersole JL, Miller CS. Salivary biomarkers of periodontal disease in response to treatment. J Clin Periodontol. 2011;38(5):434-41.

30. Gursoy UK, Kononen E, Tervahartiala T, Gursoy M, Pitkanen J, Torvi P, Suominen AL, Pussinen P, Sorsa T. Molecular forms and fragments of salivary MMP-8 in relation to periodontitis. J Clin Periodontol. 2018;45(12):1421-8.

31. Heikkinen AM, Raisanen IT, Tervahartiala T, Sorsa T. Cross-sectional analysis of risk factors for subclinical periodontitis; active matrix metalloproteinase-8 as a potential indicator in initial periodontitis in adolescents. Periodontol. 2019;90(5):484-92.

32. Morelli T, Stella M, Barros SP, Marchesan JT, Moss KL, Kim SJ, Yu N, Aspiras $M B$, Ward M, Offenbacher S. Salivary biomarkers in a biofilm overgrowth model. J Periodontol. 2014:85(12):1770-8.

33. Nascimento GG, Baelum V, Sorsa T, Tervahartiala T, Skottrup PD, Lopez R. Salivary levels of MPO, MMP-8 and TIMP-1 are associated with gingival inflammation response patterns during experimental gingivitis. Cytokine. 2019;115:135-41.

34. Verhulst MJL, Teeuw WJ, Bizzarro S, Muris J, Su N, Nicu EA, Nazmi K, Bikker $\mathrm{FJ}$, Loos BG. A rapid, non-invasive tool for periodontitis screening in a medical care setting. BMC Oral Health. 2019;19(1):87.

35. Keles Yucel ZP, Afacan B, Emingil G, Tervahartiala T, Kose T, Sorsa T. Local and systemic levels of aMMP- 8 in gingivitis and stage 3 grade $C$ periodontitis. J Periodontal Res. 2020;55(6):887-94.

36. Noack B, Kipping T, Tervahartiala T, Sorsa T, Hoffmann T, Lorenz K. Association between serum and oral matrix metalloproteinase-8 levels and periodontal health status. J Periodontal Res. 2017;52(5):824-31.

37. Sorsa T, Alassiri S, Grigoriadis A, Raisanen IT, Parnanen P, Nwhator SO, Gieselmann DR, Sakellari D. Active MMP-8 (aMMP-8) as a grading and staging biomarker in the periodontitis classification. Diagnostics (Basel). 2020;10(2):61.

38. Romero-Castro NS, Vazquez-Villamar M, Munoz-Valle JF, Reyes-Fernandez S, Serna-Radilla VO, Garcia-Arellano S, Castro-Alarcon N. Relationship between TNF-alpha, MMP-8, and MMP-9 levels in gingival crevicular fluid and the subgingival microbiota in periodontal disease. Odontology. 2020;108(1):25-33.

39. Alassiri S, Parnanen P, Rathnayake N, Johannsen G, Heikkinen AM, Lazzara R, van der Schoor P, van der Schoor JG, Tervahartiala T, Gieselmann D, et al. The ability of quantitative, specific, and sensitive point-of-care/ chair-side oral fluid immunotests for aMMP-8 to detect periodontal and Peri-implant diseases. Dis Markers. 2018;2018:1306396.

40. Kinney JS, Ramseier CA, Giannobile WV. Oral fluid-based biomarkers of alveolar bone loss in periodontitis. Ann NY Acad Sci. 2007;1098:230-51.

41. Taba M Jr, Kinney J, Kim AS, Giannobile WV. Diagnostic biomarkers for oral and periodontal diseases. Dent Clin N Am. 2005:49(3):551-71.

42. Giannobile WV, Lynch SE, Denmark RG, Paquette DW, Fiorellini JP, Williams RC. Crevicular fluid osteocalcin and pyridinoline cross-linked carboxyterminal telopeptide of type I collagen (ICTP) as markers of rapid bone turnover in periodontitis. A pilot study in beagle dogs. J Clin Periodontol. 1995;22(12):903-10

43. Payne JB, Stoner JA, Lee HM, Nummikoski PV, Reinhardt RA, Golub LM. Serum bone biomarkers and oral/systemic bone loss in humans. J Dent Res. 2011;90(6):747-51. 
44. Nazar Majeed Z, Philip K, Alabsi AM, Pushparajan S, Swaminathan D. Identification of gingival crevicular fluid sampling, analytical methods, and oral biomarkers for the diagnosis and monitoring of periodontal diseases: a systematic review. Dis Markers. 2016;2016:1804727.

45. Hassan MN, Belibasakis GN, Gumus P, Ozturk VO, Emingil G, Bostanci N. Annexin-1 as a salivary biomarker for gingivitis during pregnancy. J Periodontol. 2018;89(7):875-82.

46. Lundmark A, Hu YOO, Huss M, Johannsen G, Andersson AF, YucelLindberg T. Identification of salivary microbiota and its association with host inflammatory mediators in periodontitis. Front Cell Infect Microbiol. 2019;9:216.

47. Kc S, Wang XZ, Gallagher JE. Diagnostic sensitivity and specificity of host-derived salivary biomarkers in periodontal disease amongst adults: systematic review. J Clin Periodontol. 2020;47(3):289-308.

48. Ebersole JL, Nagarajan R, Akers D, Miller CS. Targeted salivary biomarkers for discrimination of periodontal health and disease(s). Front Cell Infect Microbiol. 2015;5:62.

49. Salminen A, Gursoy UK, Paju S, Hyvarinen K, Mantyla P, Buhlin K, Kononen E, Nieminen MS, Sorsa T, Sinisalo J, et al. Salivary biomarkers of bacterial burden, inflammatory response, and tissue destruction in periodontitis. J Clin Periodontol. 2014;41(5):442-50.

50. Gursoy UK, Kononen E, Pradhan-Palikhe P, Tervahartiala T, Pussinen PJ, Suominen-Taipale L, Sorsa T. Salivary MMP-8, TIMP-1, and ICTP as markers of advanced periodontitis. J Clin Periodontol. 2010;37(6):487-93.
51. Gursoy UK, Kononen E, Pussinen PJ, Tervahartiala T, Hyvarinen K, Suominen AL, Uitto VJ, Paju S, Sorsa T. Use of host- and bacteria-derived salivary markers in detection of periodontitis: a cumulative approach. Dis Markers. 2011;30(6):299-305.

52. Gursoy UK, Pussinen PJ, Salomaa V, Syrjalainen S, Kononen E. Cumulative use of salivary markers with an adaptive design improves detection of periodontal disease over fixed biomarker thresholds. Acta Odontol Scand. 2018;76(7):493-6.

53. Hosmer DW, Lemeshow S. Applied logistic regression. 2nd ed. New York: Wiley; 2000.

54. Afacan B, Ozturk VO, Emingil G, Kose T, Bostanci N. Alarm anti-protease trappin-2 negatively correlates with proinflammatory cytokines in patients with periodontitis. J Periodontol. 2018;89(1):58-66.

55. Miura M, Hamachi T, Fujise O, Maeda K. The prevalence and pathogenic differences of Porphyromonas gingivalis fimA genotypes in patients with aggressive periodontitis. J Periodontal Res. 2005;40(2):147-52.

56. Zhao L, Wu YF, Meng S, Yang H, OuYang YL, Zhou XD. Prevalence of fimA genotypes of Porphyromonas gingivalis and periodontal health status in Chinese adults. J Periodontal Res. 2007;42(6):511-7.

\section{Publisher's Note}

Springer Nature remains neutral with regard to jurisdictional claims in published maps and institutional affiliations.
Ready to submit your research? Choose BMC and benefit from:

- fast, convenient online submission

- thorough peer review by experienced researchers in your field

- rapid publication on acceptance

- support for research data, including large and complex data types

- gold Open Access which fosters wider collaboration and increased citations

- maximum visibility for your research: over $100 \mathrm{M}$ website views per year

At BMC, research is always in progress.

Learn more biomedcentral.com/submissions 